ERNANDO SIMIÃO DA SILVA FILHO

\title{
ORIGINALISMO AMERICANO
}

Dissertação de Mestrado

Orientador: Professor Doutor André Luiz Costa Corrêa

UNIVERSIDADE DE SÃO PAULO

FACULDADE DE DIREITO

São Paulo-SP

2020 
ERNANDO SIMIÃO DA SILVA FILHO

\section{ORIGINALISMO AMERICANO}

Dissertação apresentada à Banca Examinadora do Programa de Pós-Graduação em Direito, da Faculdade de Direito da Universidade de São Paulo, como exigência parcial para obtenção do título de Mestre na área de concentração em Direito do Estado, sob orientação do Professor Doutor André Luiz Costa Corrêa

UNIVERSIDADE DE SÃO PAULO

FACULDADE DE DIREITO

São Paulo-SP

2020 


\section{ORIGINALISMO AMERICANO}

Dissertação apresentada à Banca Examinadora do Programa de Pós-Graduação em Direito, da Faculdade de Direito da Universidade de São Paulo, como exigência parcial para obtenção do título de Mestre na área de concentração em Direito do Estado, sob orientação do Professor Doutor André Luiz Costa Corrêa

Aprovado em:

Banca Examinadora

Prof. Dr.

Instituição:

Julgamento:

Assinatura:

Prof. Dr. Instituição:

Julgamento: Assinatura:

Prof. Dr. Instituição:

Julgamento:

Assinatura:

Prof. Dr. Instituição:

Julgamento: Assinatura: 


\section{RESUMO}

SILVA FILHO, Ernando Simião da. Originalismo Americano. 2020. 132f. Dissertação (Mestrado) - Faculdade de Direito, Universidade de São Paulo, São Paulo, 2020.

Neste trabalho é estudada e exposta, através de pesquisa bibliográfica em fontes preponderantemente americanas, a escola de interpretação constitucional americana chamada originalismo. Inicialmente, é elaborado um panorama geral, abordando a evolução histórica do originalismo nos Estados Unidos como reação ao ativismo judicial da Suprema Corte, comentando-se as principais decisões paradigmáticas. Após, faz-se uma exposição das correntes em que se divide, principalmente o intencionalismo e o textualismo, expondo-se as teses de Robert Bork, Raoul Berger e Antonin Scalia, bem como de outros autores que conformam o panorama atual destas doutrinas. Na terceira parte, são analisadas as objeções dos principais críticos, no ambiente americano, do originalismo, como Paul Brest, H. Jefferson Powell e David Strauss. Na conclusão retomam-se os temas expostos e discute-se a possibilidade de que o originalismo possa, de alguma forma, contribuir para a atividade judicial no Brasil.

Palavras-chave: EUA. Direito Constitucional. Interpretação Constitucional. Hermenêutica Jurídica. Constituição Americana. Originalismo. Intencionalismo. Textualismo. 


\begin{abstract}
SILVA FILHO, Ernando Simião da. American Originalism. 2020. 132p. Dissertation (Master Degree) - Faculdade de Direito, Universidade de São Paulo, São Paulo, 2020.

In this work, through bibliographic research in prevailent american sources, the american school of constitutional interpretation called originalismo is studied and exposed. Initially, a general view is elaborated, expounding the historic evolution of originalism in the United States as reaction to judicial activism of the Supreme Court, remarking the main paradigmatic decisions. After, it's divisions are exposed, mainly intencionalism and textualism, through the ideas of Robert Bork, Raoul Berger and Antonin Scalia, and another authors that conform the actual landscape of these doctrines. On third part, the principal objections of originalism of the leadins critics are analised, in american environment, like Paul Brest, H. Jefferson Powell and David Strauss. In conclusion, the studied themes are reviewed and the possibility of the contribution of some type of the originalismo to judicial activity in Brazil are discussed.
\end{abstract}

Keywords: USA. Constitutional Law. Constitutional Interpretation. Hermeneutics. American Constitution. Originalism. Intencionalism. Textualism. 
Catalogação da Publicação

Serviço de Biblioteca e Documentação

Faculdade de Direito da Universidade de São Paulo

SILVA FILHO, ERNANDO SIMIÃO DA

ORIGINALISMO AMERICANO ; ERNANDO SIMIÃO DA SILVA

FILHO ; orientador ANDRÉ LUIZ COSTA CORREA -- São Paulo, 2020

132

Dissertação (Mestrado - Programa de Pós-Graduação em Direito do Estado) - Faculdade de Direito,

Universidade de São Paulo, 2020.

1. DIREITO CONSTITUCIONAL. 2. INTERPRETAÇÃO

CONSTITUCIONAL. 3. HERMENÊUTICA JURÍDICA. 4

CONSTITUIÇÃO AMERICANA. 5. ORIGINALISMO. I. CORRÊA, ANDRÉ LUIZ COSTA, orient. II. Título. 


\section{DEDICATÓRIA}

Aos meus pais, Edinete e Ernando (in memorian).

À minha esposa, Alene, e aos nossos filhos, Luísa, Cecília e Ernando Neto. 


\section{AGRADECIMENTOS}

À Procuradoria Geral do Estado do Amazonas, pelo apoio financeiro e institucional.

Ao Professor Doutor André Luiz Costa Corrêa, exemplo intelectual e moral, sem cuja orientação tal trabalho não teria sido finalizado.

Ao Centro Integrado de Ensino Superior do Amazonas - CIESA, pela iniciativa inédita de cultivar os estudos de pós-graduação no Estado do Amazonas.

À Faculdade de Direito da Universidade de São Paulo, pela nobre e desprendida ação de trazer seu mestrado à Região Norte do Brasil. 
It is simply not compatible with democratic theory that laws mean whatever they ought to mean, and that unelected judges decide what that is.

(Antonin Scalia) 
SUMÁRIO

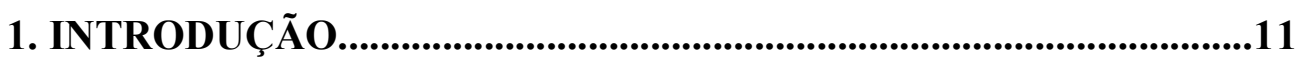

2. ESCORÇO HISTÓRICO....................................................................17

3. INTENCIONALISMO....................................................................

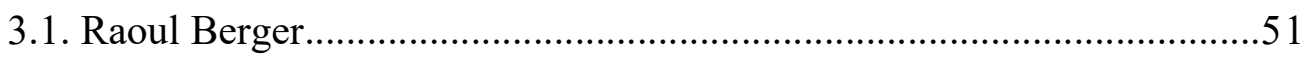

3.2. Robert Bork.............................................68

4. TEXTUALISMO.................................................81

5. PODERAÇÕES CRÍTICAS AO ORIGINALISMO.............................102

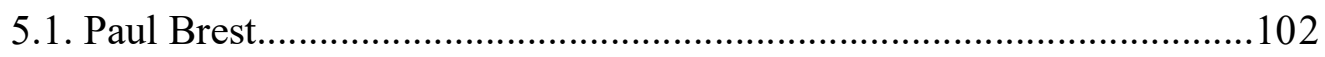

5.2. H. Jefferson Powell......................................... 105

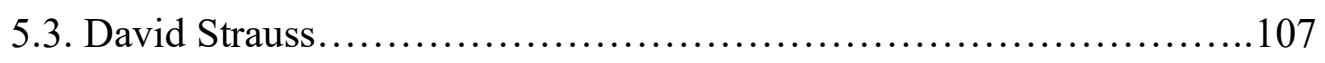

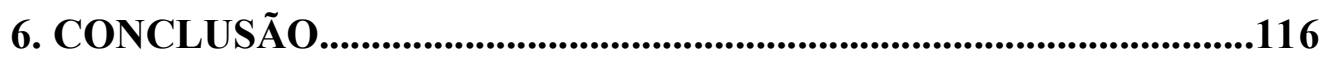

7. REFERENCIAS..........................................................................120 


\section{INTRODUÇÃO}

Por originalismo compreende-se o movimento hermenêutico constitucional, pouco organizado, que surgiu na década de 1970 nos Estados Unidos, sendo formado por diversas vertentes que, não raro, divergem entre si. Sua principal propriedade encontrase no fato de seus adeptos defenderem que o significado do texto da Constituição deve ser o mesmo da época em que foi editada.

Um dos principais objetivos dos originalistas é a contenção da atividade judicial legislativa e administrativa na interpretação da constituição, conforme pode ser depreendido da seguinte passagem de Randy E. Barnett:

A constituição é uma lei projetada para restringir os legisladores. Embora a própria Constituição possa ter múltiplos propósitos e funções, sua característica de ser escrita tem poucos. Não obstante seja inteiramente possível haver constituições não escritas, constituições escritas são escritas por uma razão. Primeiramente, constituições são estabelecidas em formato escrito para melhor constranger os atores políticos imbuídos de poderes para a consecução de diversos fins públicos. Em particular, ela é escrita para que tais atores não possam eles mesmos fazer as leis pelas quais são regidos. ${ }^{1}$ (tradução livre)

Tal movimento caracteriza-se por uma tentativa de alguns juristas americanos de estabelecer que a interpretação da Constituição Americana deve ser efetuada a partir do significado público do texto normativo fixado na época em que foi escrito. Indigitado significado será construído com base na intenção dos constituintes (originalismo de tendência intencionalista) ou com espeque no sentido público das palavras utilizadas no tempo da promulgação (originalismo de tendência textualista). Corroborando tal entendimento, colaciona-se o escólio de Lawrence B. Solum:

(...) A história revela que a teoria originalista contemporânea evoluiu as principais correntes do originalismo começam com uma ênfase nas intenções originais dos constituintes, mas tem-se movido gradualmente para a visão de que o "significado original" da Constituição é o "sentido

\footnotetext{
1 "The Constitution is a law designed to restrict the lawmakers. Although the Constitution itself may have multiple purposes and functions, its "writtenness" has many fewer. Though it is entirely possible to have na unwritten constitution, written constitutions are in writing for a reason. Primarily, constitutions are put in writing to better constrain the political actors it empowers to accomplish various ends. In particular, it is put in writing so these actors cannot themselves make the laws by which they make law." (BARNETT, Randy E.. Restoring the Lost Constitution: The Presumption of Liberty. Princeton: Princeton University Press, 2004. p. 103)
} 
público original” do texto. ${ }^{2}$ (tradução livre)

Uma vez fixado tal significado, deve-se utilizá-lo nas aplicações aos casos concretos posteriores, ainda que decorridos séculos após a publicação do texto, até que a Constituição seja alterada pelas vias legitimamente cabíveis. Assim, percebe-se que um dos objetivos do originalismo, que seus adeptos confessam abertamente, é de certa forma tolher o poder discricionário do magistrado na aplicação dos textos normativos na atividade judicial. Neste sentido, aduz Raoul Berger que

(...) antiativistas (originalistas) sustentam que juízes não estão autorizados a revisar a Constituição e que ela deve ser interpretada à luz das explanações dos Founders sobre o que eles pretendiam dizer, nem mais, nem menos. ${ }^{3}$ (tradução livre)

Ressalte-se, ademais, que há diferença entre significado do texto e aplicação ao caso concreto, problema que será trabalhado no capítulo seguinte.

Existem diversas correntes originalistas, com muitas discrepâncias entre si, fazendo com que se chegue até a questionar-se se é possível falar-se em originalismo, por falta de pontos em comum. Todavia, conforme o ensino de Lawrence B. Solum, há dois pontos em comum, duas teses fundamentais sobre as quais todo jurista que se confessa originalista não ousa discordar, a tese da fixação (the fixation thesis) e a tese da contribuição (the contribution thesis).

Segundo Lawrence B Solum, a tese da fixação (the fixation thesis) consiste em concordar que o significado original da constituição é fixado no momento da elaboração do texto ${ }^{4}$. Já a tese da contribuição (the contribution thesis) é entendida como a ideia de que o significado original da constituição deve exercer um papel fundamental na doutrina

\footnotetext{
2 "The history reveals that contemporary originalist theory has evolved - the mainstream of originalist theory began with an emphasis on the original intentions of the framers but has gradually moved to the view that the "original meaning" of the constitution is the "original public meaning" of the text." (SOLUM, Lawrence B. What is Originalism? The Evolution of Contemporary Originalist Theory, in HUSCROFT, David; MILLER, Bradley W. (Org.). The Challenge of Originalism. $1^{\text {a }}$ ed. New York: Cambridge University Press, 2011. p. 12)

3 "antiactivists (originalists) maintain that judges are not authorized to revise the Constitution 16 and that it is to be construed in light of the Founders' explanations of what they meant to accomplish, no more, no less." (BERGER, Raoul. Government by Judiciary - The Transformation of the Fourteenth Amendment. $2^{\mathrm{a}}$ ed. Indianápolis: Liberty Fund, 1997. pp. 21-22)
}

${ }^{4}$ SOLUM, Lawrence B. Op. cit. p. 33. 
do direito constitucional ${ }^{5}$. Em suma, o indigitado autor defende que

No domínio da teoria constitucional, originalismo é uma família de visões que se agrupam em torno de duas ideias centrais: a tese da fixação e a tese da contribuição. Todos ou praticamente todos os originalistas concordam que o sentido original da Constituição foi fixado no momento em que cada provisão foi elaborada e ratificada. Também, quase todos os originalistas concordam que o sentido original deve exercer uma função importante no conteúdo da doutrina constitucional: a maioria dos originalistas concorda que as cortes devem ver a si mesmas como restringidas pelo sentido original e que deve haver razões muito fortes para que seja legítimo afastar-se dele. ${ }^{6}$ (tradução livre)

De mais a mais, debates sobre a escola de interpretação constitucional denominada originalismo têm sido o foco central da teoria jurídica contemporânea nos Estados Unidos nas últimas décadas, não obstante a majoritária falta de interesse no meio acadêmico brasileiro em relação ao tema.

Talvez tal apatia dê-se em virtude das diferenças jurídicas, culturais e políticas entre Brasil e Estados Unidos. A longa tradição democrática e de estabilidade institucional dos Estados Unidos, país nascido de uma revolução republicana inédita na história e cuja constituição escrita já dura mais de duzentos anos, tendo sido submetida a poucas emendas em termos relativos, despertou o interesse das instituições e dos estudiosos para a necessidade de interpretação dos textos normativos de forma a transmitir segurança jurídica aos cidadãos.

Todavia, apesar da crítica de Luís Roberto Barroso no sentido de que o originalismo é uma corrente de pensamento ultrapassada ${ }^{7}$, em face do desenvolvimento hermenêutico do neoconstitucionalismo dos dias atuais, tal assertiva merece ser lida cum grano salis.

\footnotetext{
${ }^{5}$ Idem, p. 35.

6 "Within the domain of constitutional Theory, originalismo is a Family of views that cluster around two central ideas: the fixation thesis and the contribution thesis. All or almost all originalists agree that the original meaning of the Constitution was fixed at the time each provision was framed and ratified. Almost all originalists agree that original meaning must make na importante contribution to the contente of constitutionla doctrine: Most originalists agree that courts should view themselves as constrained by original meaning and that very good reasons are required for legitimate departures form that constraint.". Idem, pp. 35-36.
}

${ }^{7}$ BARROSO, Luís Roberto. Interpretação e Aplicação da Constituição. $7^{a}$ ed. São Paulo: Saraiva, 2009, pp. 116-119. 
No Brasil, embora o originalismo não seja objeto de estudos e seus métodos não encontrem eco no ambiente acadêmico ou judicial, algumas técnicas originalistas são, vez ou outra, adotadas em julgamentos importantes do Supremo Tribunal Federal. Por exemplo $^{8}$, no julgamento da ADPF n. 153, em que se pretendia uma reinterpretação da Lei de Anistia, uma solução tipicamente originalista foi adotada pela Corte para desfecho do caso.

No mesmo diapasão, quando o Supremo determinou qual o conceito de veículo automotor, para fins de incidência do IPVA, no Recurso Extraordinário n. 134.509-8, foi indispensável pesquisar os antecedentes históricos, inclusive com consulta às atas da Constituinte de 1988. E, dentre outros casos, quando do julgamento do Habeas Corpus $n$. 82.424/RS, o Relator fez uso da interpretação originalista no seu voto, transcrevendo, inclusive, o discurso feito pelo autor da emenda na Assembleia Nacional Constituinte em 03/02/1988.

Tais julgados indicam que de quando em quando o Supremo Tribunal Federal adota alguma técnica tipicamente originalista na resolução de conflitos hermenêuticos, frequentemente quando a orientação ideológica do constituinte originário vai ao encontro da ideologia do ministro relator ou da construção da maioria do plenário. É um verdadeiro exercício de adivinhação tentar prever qual a técnica de interpretação que a Suprema Corte do Brasil irá empregar em cada um dos seus julgados.

A rigor, o originalismo, conforme o escólio de Ilan Wurman, tem fulcro na forma mais natural de interpretar-se um texto, qual seja, conforme o significado das palavras utilizadas na época em que foram escritas. Se este método é o mais adequado para a aplicação dos textos constitucionais ou legais, isso é outra questão que será também analisada. Ensina o referido autor:

(...) Quando o originalismo não era sequer algo? Originalismo é totalmente uma ideia do senso comum, depois de tudo. Ele possui várias matizes, mas nós podemos defini-lo superficialmente como a ideia de que a Constituição deveria ser interpretada como suas palavras eram originalmente entendidas pelos Constituintes que escreveram a Constituição em 1787 e pelo povo que a ratificou entre 1787 e 1789. De forma um pouco mais ampla, é a ideia de que palavras têm um significado público original ao tempo em que foram faladas ou escritas

\footnotetext{
${ }^{8}$ Exemplos retirados de MORALES, Cesar Mecchi. Originalismo e Interpretação Constitucional. (Tese de Doutorado). São Paulo, 2011. Disponível em http://www.teses.usp.br/teses/disponiveis/2/2134/tde16042012-161140/pt-br.php, acessado em 12/12/2016.
} 
e apresentadas ao mundo. ${ }^{9}$ (tradução livre)

Os problemas práticos que fizeram surgir o originalismo na América são antigos e contemporâneos ao próprio advento da Constituição Americana, no final do Século XVIII, tais como a questão da normatividade e da supremacia da Constituição, o controle das normas infraconstitucionais e o advento do ativismo judicial da Suprema Corte Americana. Essas questões estão presentes na cultura constitucional desse país desde a promulgação da constituição até o presente.

Em outros termos, pode-se dizer que a questão da normatividade e da supremacia da Constituição e o controle das normas infraconstitucionais são causas remotas do originalismo, enquanto o ativismo judicial da Suprema Corte Americana, mormente no Século XX, é a principal causa próxima desse movimento.

Serão analisadas algumas decisões paradigmáticas e suas implicações para a formação do ambiente jurídico que possibilitou o nascimento do originalismo, como, verbi gratia, Marbury v. Madison, McCulloch v. Maryland, Dred Scott vs. Sandford, Lochner vs. New York, Brown vs. Board of Education e Roe vs. Wade.

Será abordada, então, o que se convencionou chamar de Era Warren-Burguer, por causa da presidência dos Chief Justices Earl Warren (1954 - 1969) e Warren E. Burger (1969 - 1986), época marcada por um ativismo judicial da Suprema Corte inédito. Esse ativismo gerou inevitavelmente uma reação conservadora nos âmbitos acadêmico, político e judicial, que foi a causa imediata do movimento que aqui se pretende estudar.

Dar-se-á um panorama geral das vertentes do originalismo surgidas a partir dos anos 1970, analisando-se do intencionalismo de Raoul Berger e Robert Bork ao textualismo de Antonin Scalia e outros autores, ressaltando a importância que o debate entre originalistas e não-originalistas tem nos dias de hoje.

O tema merece ainda mais destaque pelas recentes e futuras nomeações para a Suprema Corte, em função da posse de Donald Trump no cargo de Presidente dos Estados Unidos. Este presidente prometeu que um dos requisitos para a escolha de um candidato

\footnotetext{
9 “(...) When was originalismo ever not a thing? Originalism is quite a commonsensical idea, after all. It has many flavors, but we can define it broadly as the idea that the Constitution should be interpreted as its words were originally understood by the Framers who wrote the Constitution in 1787 and by the public that ratified it between 1787 and 1789. More broadly still, it is the idea that words have an original public meaning at the time they were spoken or written and presented to the world." (WURMAN, Ilan. A Debt Against the Living - An Introduction to Originalism. Cambridge: Cambridge University Press, 2017. p. 11)C
} 
à Suprema Corte é ser ele originalista.

Em capítulo próprio, será feita uma análise das objeções dos principais críticos, no ambiente americano, do originalismo, como Paul Brest, H. Jefferson Powell e David Strauss.

Por fim, serão retomados os temas expostos e discutida a possibilidade de que o originalismo possa, de alguma forma, contribuir para a atividade judicial no Brasil. Questões envolvendo segurança jurídica, separação de poderes, limites do ativismo judicial e legitimidade do Poder Judiciário para inovar no ordenamento jurídico são universais para todos os países democráticos que são regidos por constituições escritas. 


\section{CONCLUSÃO}

Foram expostos aqui os principais delineamentos da escola de interpretação constitucional chamada originalismo, a qual se divide em algumas vertentes bem díspares entre si, havendo quem diga haver não o originalismo, mas vários originalismos ${ }^{252}$.

Entrementes, como visto, há pelo menos duas características fundamentais, apontadas por Lawrence Solum ${ }^{253}$, que estão presentes em qualquer teoria que possa ser classificada como originalista. São elas a tese da fixação (the fixation thesis) e a tese da contribuição (the contribution thesis). A assertiva de Solum foi aceita pelos teóricos, pelo que Ian Bartrum ${ }^{254}$, por exemplo, chegue a classificar as duas teses de dogmas do originalismo.

A tese da fixação (the fixation thesis) traduz-se na ideia de que o significado do texto normativo é estabelecido ao tempo da promulgação, sendo este significado baseado na intenção do legislador ou na interpretação puramente textual das palavras, como entendidas à época. De caráter temporal, este é o principal dogma originalista. Sem ele, não há que se falar, absolutamente, em originalismo.

A tese da contribuição (the contribution thesis) estriba-se na noção de que o significado original da Constituição, estabelecido conforme a tese da fixação, deve exercer o papel central na ciência do direito constitucional. Este postulado vai de encontro ao conceito de emanações das penumbras normativas, tais como idealizadas pelo Justice William O. Douglas em Griswold v. Connecticut (1965).

Explicitados os dois dogmas presentes em qualquer movimento originalistas, foi efetuada uma breve digressão histórica de várias decisões da Suprema Corte que influenciaram, ainda que tardiamente, o advento do originalismo, como, por exemplo, Marbury v. Madison, McCulloch v. Maryland, Dred Scott vs. Sandford, Lochner vs. New

\footnotetext{
${ }^{252}$ SOLUM, Lawrence B.. What is Originalism? The Evolution of Contemporary Originalist Theory, in HUSCROFT, David; MILLER, Bradley W. (Org.). The Challenge of Originalism. $1^{\mathrm{a}}$ ed. New York: Cambridge University Press, 2011. pp. 32-33.

${ }^{253}$ Idem. pp. 35-36.

${ }^{254}$ BARTRUM, Ian. Two Dogmas of Originalism. Washington University Jurisprudence Review, v. 7, n. 2, 2015.2 Disponível em https://openscholarship.wustl.edu/cgi/viewcontent.cgi?article=1120\&context=law jurisprudence, acessado em 04.08.2020.
} 
York, Brown vs. Board of Education e Roe vs. Wade.

As duas principais vertentes do originalismo são o intencionalismo e o textualismo. O primeiro, em que o fator fundamental para estabelecimento do significado original da constituição é a intenção do constituinte, surgiu nos anos de 1970 e teve como principais ícones Raoul Berger e Robert Bork.

O textualismo, em que o centro da atividade interpretativa se deslocou da intenção dos Framers para o significado público objetivo do texto, à época da promulgação, teve como seu primeiro e grande promotor, na academia e no âmbito judicial, principalmente, Antonin Scalia. O textualismo hoje é a vertente dominante, mas ainda há uns poucos autores $^{255}$ em defesa da matiz intencionalista.

Trabalhou-se sobre as ideias principais de Raoul Berger, Robert Bork e Antonin Scalia, trilhando-se o caminho temporal do intencionalismo ao textualismo, sempre transpassando as ideias expostas com as opiniões de outros autores, antigos e contemporâneos.

De mais a mais, como o originalismo rechaça o conceito de mutação constitucional, fez aqui uma breve exposição da válvula de escape originalista para o problema da incidência de normas antigas sobre situações novas e imprevistas pelo legislador originário. Tal solução descansa na diferenciação entre interpretação do sentido e aplicação da norma, dois momentos distintos da atividade jurídica, desenvolvida por Christopher R. Green ${ }^{256}$ e comentada por Ilan Wurman em A Debt Against the Living ${ }^{257}$.

Como todo estudo que pretenda expor alguma teoria por diversos prismas, com intuito de melhor apreender o objeto de estudo, foram exibidas, em capítulo próprio, algumas objeções ao originalismo dos principais críticos, no ambiente americano, como Paul Brest, H. Jefferson Powell e David Strauss.

\footnotetext{
255 Por exemplo: ALEXANDER, Larry. Connecting the Rule of Recognition and Intentionalist Interpretation: An Essay in Honor of Richard Kay. San Diego Legal Studies Paper n. 19, (2019). Disponível em https://papers.ssrn.com/sol3/papers.cfm?abstract id=3448695, acessado em 04.08.2020.

${ }^{256}$ GREEN, Christopher R., Originalism and the Sense-Reference Distinction. St. Louis University Law Journal, V. 50, 2006. Disponível em https://ssrn.com/abstract=798466, acessado em 18.06.2020.

257 WURMAN, Ilan. A Debt Against the Living - An Introduction to Originalism. Cambridge: Cambridge University Press, 2017. p. 11.
} 
Deu-se maior relevo à obra de David Strauss, The Living Constitution ${ }^{258}$, em função dos fortes argumentos ali esposados. Com efeito, o principal problema do originalismo, que ainda se encontra em aberto, sem solução satisfatória, é o Problema de Jefferson (Jefferson Problem's).

Os originalistas americanos têm a difícil missão de encontrar uma justificativa legítima e convincente para que a mão dos mortos do passado (dead hand of the past) distante continue a governar a geração presente, que não participou da elaboração da Lei Fundamental do país.

Espera-se que tenha sido cumprido o desiderato deste trabalho, qual seja, fazer uma breve exposição em língua pátria da escola de interpretação constitucional americana originalismo. É possível especular-se se o originalismo poderia ser aplicado no Brasil, principalmente no tempo atual, em que o Supremo Tribunal Federal assumiu um papel protagonista na implementação de políticas públicas.

Tal especulação foge ao escopo desta dissertação. Mas, não deixa de ser interessante a reflexão. Nos Estados Unidos, o principal obstáculo a ser transposto pelo originalismo para ser aceito amplamente como método legitimo de interpretação é o Problema de Jefferson, agravado pelo dificultoso, quase impossível, procedimento de emendas à constituição daquele país.

Repita-se, conforme o artigo V da Constituição Americana, somente por iniciativa de dois terços dos membros da Câmara e do Senado ou a pedido de dois terços das legislaturas dos 50 Estados (33 dos 50 Estados), poderá ser emendada a Constituição e, para a emenda entrar em vigor, é necessário que seu texto seja ratificado por três quartos dos Estados.

Ou seja, nos Estados Unidos a constituição foi escrita em sua maior parte há mais de dois séculos e o processo de emenda é extremamente difícil, o que provoca um certo engessamento da constituição.

Porém, o Brasil não enfrenta tais obstáculos. Não há o Problema de Jefferson aqui, como lá. A Constituição do Brasil é relativamente nova, tendo sido promulgada em 1988, e já foi emendada mais de cem vezes. Ademais, como é de se concluir, o procedimento de aprovação de uma emenda constitucional no Brasil é relativamente simples,

${ }^{258}$ STRAUSS, David A.. The Living Constitution. New York: Oxford University Press, 2010. 
demandando apenas a aprovação de ambas as casas legislativas federais por três quintos dos votos, em dois turnos de votação, sem necessidade de consulta aos Estados.

Portanto, é questão interessante o estudo da legitimidade do Supremo Tribunal Federal para alterar ao seu talante os sentidos das normas insculpidas nos textos constitucionais, posto que aqui não há o Problema de Jefferson. O originalismo nas terras brasileiras poderia, talvez, servir de fundamento para algum plano de limitação da atividade legiferante, através do ativismo judicial, da Corte Suprema.

Segurança jurídica, separação de poderes, limites e legitimidade do Poder Judiciário para inovar no ordenamento jurídico de um país, em que a constituição garante a renovação de seu texto com relativa facilidade, são temas por excelência a serem gizados por algum jurista sob a metodologia do originalismo. Todavia, como dito, tal pesquisa foge ao escopo desta dissertação, que era a exposição do originalismo nos Estados Unidos, podendo vir a ser objeto de trabalho do missivista em eventual empreitada futura. 


\section{REFERÊNCIAS}

ALEXANDER, Larry. Connecting the Rule of Recognition and Intentionalist Interpretation: An Essay in Honor of Richard Kay. San Diego Legal Studies Paper n. 19, (2019). Disponível em https://papers.ssrn.com/sol3/papers.cfm?abstract id=3448695, acessado em 04.08.2020. BALKIN, Jack M. Abortion and Original Meaning. Faculty Scholarship Series. Paper 228, 2007. Disponível em http://digitalcommons.law.yale.edu/fss_papers/228 (acessado em 24/01/2017).

. Clarence Thomas's Originalism. Balkinization, 2007. Disponível em https://balkin.blogspot.com/2007/07/clarence-thomass-originalism.html (acessado em 04/01/2019).

. Framework Originalism and the Living Constitution. Northwestern University Law Review, 2009. Disponível em https://ssrn.com/abstract=1290869 (acessado em 24/01/2017).

. Living Originalism. Cambridge: Harvard University Press, 2011.

. Original Meaning and Constitutional Redemption. Constitutional Commentary, Vol. 24, 2007; Yale Law School, Public Law Working Paper No. 140. Disponível em https://ssrn.com/abstract=987060 (acessado em 24/01/2017).

. The New Originalism and the Uses of History. Fordham Law Review, Forthcoming; Yale Law School, Public Law Working Paper No. 303, 2013. Disponível em https://ssrn.com/abstract=2303980 (acessado em 24/01/2017).

. Why are Americans Originalist? Yale Law School, Public Law Research Paper No. 492, 2014. Disponível em https://ssrn.com/abstract=2379587 (acessado em 24/01/2017).

BARBER, Sotirius A.; FLEMING, James E. Constitutional Interpretation: The Basic Questions. 1a ed. Oxford: Oxford University Press, 2007.

BARNETT, Randy E. An Originalism for Nonoriginalists. The University of Chicago Law Review, VOLUME 56 NUMBER 4 FALL, 1989. Disponível em http://scholarship.law.georgetown.edu/cgi/viewcontent.cgi?article=2243\&context=facp ub (acessado em 24/01/2017). 
. Interpretation and Construction. Harvard Journal of Law and Public

Policy, Vol. 34, 2011; Georgetown Public Law Research Paper No. 12-034, 2012. Disponível em https://ssrn.com/abstract=2021318 (acessado em 24/01/2017).

. Restoring the Lost Constitution: The Presumption of Liberty. Princeton: Princeton University Press, 2004.

. Scalia's Infidelity: A Critique of Faint-Hearted Originalism. University of Cincinnati Law Review, Vol. 75, No. 7, 2006; Boston University School of Law Working Paper No. 06-01; Georgetown Public Law Research Paper No. 880112. Disponível em https://ssrn.com/abstract=880112 (acessado em 24/01/2017).

. The Original Meaning of the Judicial Power. Boston Univ. School of Law Working Paper No. 03-18, 2003. Disponível em https://ssrn.com/abstract=437040 (acessado em 24/01/2017).

BARNETT, Randy E; BLACKMAN, Josh. An Introduction to Constitutional Law. New York: Wolters Kluwer, 2020.

BARNETT, Randy E; BERNICK, Evan. The Letter and the Spirit: A Unified Theory of Originalism. Georgetown Law Faculty Publications and Other Works, 107 Geo. L.J. 1, 2018. Disponível em https://scholarship.law.georgetown.edu/facpub/2000 (acessado em 24/01/2020).

BARROSO, Luís Roberto. Direito Constitucional Contemporâneo. $3^{\mathrm{a}}$ ed. São Paulo: Saraiva, 2011.

. Interpretação e Aplicação da Constituição. $7^{\mathrm{a}}$ ed. São Paulo: Saraiva, 2009.

BARTRUM, Ian. Two Dogmas of Originalism. Washington University Jurisprudence Review, v. $7, \quad$ n. 2 , 2015. Disponível em https://openscholarship.wustl.edu/cgi/viewcontent.cgi?article $=1120 \&$ context $=1$ law juris prudence, acessado em 04.08.2020.

BAUM, Lawrence. The Supreme Court. $11^{\mathrm{a}}$ ed. Thousand Oaks: CQ Press College, 2012.

BELTRÁN DE FELIPE, Miguel. Originalismo e Interpretación - Dworkin vs. Bork: una polémica constitucional. Madri: Editorial Civitas, 1989. 
BERGER, Raoul. Government by Judiciary - The Transformation of the Fourteenth Amendment. $2^{\mathrm{a}}$ ed. Indianápolis: Liberty Fund, 1997.

. Congress v. The Supreme Court. Cambridge: Harvard University Press, 1969.

. Impeachment: the Constitutional Problems. Boston: Harvard University Press, 1973.

. Paul Brest's Brief for an Imperial Judiciary. Maryland Law Review, v. 40, 1981. Disponível

em https://digitalcommons.law.umaryland.edu/cgi/viewcontent.cgi? article=2437\&context=

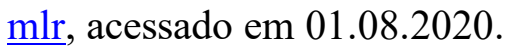

Reflections on Constitutional Interpretation. Brigham Young University

Law $\quad$ Review, $1997 . \quad$ Disponível em https://digitalcommons.law.byu.edu/lawreview/vol1997/iss3/1 $\quad$ (acessado em 05/02/2020).

. The Activist Legacy of the New Deal Court. Washington Law Review 751, 1984. Disponível em https://digitalcommons.law.uw.edu/wlr/vol59/iss4/4. (acessado em 03.03.2020).

. The President, Congress, and the Courts. Yale Law Journal, 1974, disponível em https://digitalcommons.law.yale.edu/ylj/vol83/iss6/1, acessado em 05.03.2020.

. Constructive Contempt: A Post Mortem. University of Chicago Law Review 9, $1942 . \quad$ Disponível em https://chicagounbound.uchicago.edu/uclrev/vol9/iss4/5/, acessado em 05.03.2020.

. Paul Brest's Brief for an Imperial Judiciary. Maryland Law Review, 40, 1981, disponível em https://digitalcommons.law.umaryland.edu/mlr/vol40/iss1/4, acessado em 05.06.2020.

BERMAN, Mitchell N., Originalism is Bunk. New York University Law Review, V. 84 april 2009 N. 1. Disponível em https://ssrn.com/abstract=1078933 (acessado em 03/02/2017).

BERNS, Walter. Democracy and the Constitution. $1^{\text {a }}$ ed. Washington (DC): Aei Press, 2006. 
BICKEL, Alexander. The Least Dangerous Branch: The Supreme Court at the Bar of Politics. $2^{\mathrm{a}}$ ed. New Haven: Yale University Press, 1986.

. The Original Understanding and the Segregation Decision. Harvard Law Review, V. 69, n. 1, 1955. Disponível em https://digitalcommons.law.yale.edu/cgi/viewcontent.cgi? article $=4958 \&$ context $=$ fss_pa pers (acessado em 03/02/2017)..

BLACK, Henry Campbell. Black's Law Dictionary. $5^{\text {a }}$ ed. St. Paul Minn: West Publishing, 1979.

BOOHER, Troy L., Putting Meaning in Its Place: Originalism and Philosophy of Language. Law \& Philosophy, July 2006. Disponível em https://ssrn.com/abstract=901540 (acessado em 03/02/2017).

BORK, Robert. Coercing Virtue: the worldwide rule of judges. Washington: American Enterprise Institute, 2003.

Commentary: The Impossibility of Finding Welfare Rights in the Constitution. Washington University Law Quarterly, 695, 1979. Disponível em https://openscholarship.wustl.edu/law lawreview/vol1979/iss3/3, acessado em 02.07.2020.

. Tradition and Morality in Constitutional Law. 1984. Disponível em https://www.aei.org/research-products/speech/tradition-and-morality-in-constitutionallaw/, acessado em 05.07.2020.

. A Country I Do Not Recognize: The Legal Assault on American Values. $1^{\mathrm{o}}$ ed. Stanford(CA): Hoover Institution, 2005.

. Neutral Principles and Some First Amendment Problems. Indiana Law Journal, v. 47, 1971.

. The Antitrust Paradox. 1ª ed. New York: Basic Books, 1978.

. The Tempting of America. $1^{\text {a }}$ ed. New York: Touchstone, 1991.

. A Time to Speak: Selected Writings and Arguments. Wilmington: Intercollegiate Studies Institute, 2008.

BRENNAN, William J.. Speech to the Text and Teaching Symposium, in: CALABRESI, Steven G. (org.). Originalism - A Quarter-Century of Debate. 
Washington: Regnery, 2007. pp. 55-70.

BREST, Paul. The Misconceived Quest for Original Understanding. Boston

University Law Review, vol. 60, n. 2. Disponível em https://heinonline.org/HOL/Page?lname $=\&$ public $=$ false $\&$ collection=journals $\&$ handle $=\mathrm{h}$ ein.journals/bulr60\&men hide $=$ false \&men $\_$tab $=$toc\&kind $=\&$ page $=204$, acessado em 05.07.2019.

BRISON, Susan J; SINNOTT-ARMSTRONG, Walter (org). Contemporary Perspectives on Constitutional Interpretation. 1ª ed. Berkeley: Westview Press, 1993.

CALABRESI, Steven G. (org.). Originalism - A Quarter-Century of Debate. Washington: Regnery, 2007.

. Originalism and James Bradley Thayer. Northwestern University Law Review, v. 113, n. 6, 2019.

CANOTILHO, José Joaquim Gomes. Direito Constitucional e Teoria da Constituição. $7^{\mathrm{a}}$ ed. Coimbra: Almedina, 2003.

CASTRO, Carlos Roberto Siqueira. O Devido Processo Legal e os Princípios da Razoabilidade e da Proporcionalidade. 3a . ed. Rio de Janeiro: Forense, 2005.

COOLEY, Thomas M. A Treatise on the Constitutional Limitations Which Rest upon the Legislative Power of the States of the American Union. $2^{\mathrm{a}}$ ed. Boston: Little, Brown \& Company, 1871.

CORRÊA, André Luiz Costa. Crítica à desconsideração do campo objetivo dos pontos-limites de reconhecimento quando da transformação autopoiética destes em enunciados jurídicos pelos agentes-comunicantes legítimos - implicações cibernéticas da determinação do conteúdo (significado) e do sentido (significação) dos pontos-limites de reconhecimento para a transformação autopoiética das normas jurídicas no sistema jurídico brasileiro. Tese de Doutorado, Pontifícia Universidade Católica de São Paulo, 2011.

CROSS, Franklin. The Failed Promise of Originalism. $1^{\text {a }}$ ed. Stanford: Stanford Law Books, 2013.

DWORKIN, Ronald. Taking Rights Seriously. Cambridge: Harvard University Press, 1997. 
. A Matter of Principle. Oxford: Oxford University Press, 1985.

. Social Rules and Legal Theory. Yale Law Journal, 81, 1972. Disponível em https://digitalcommons.law.yale.edu/ylj/vol81/iss5/3/ (acessado em 05/02/2020).

ELY, John Hart. Democracy and Distrust - A Theory of Judicial Review. Cambridge: Harvard University Press, 1980.

EPSTEIN, Lee; WALK, Thomas G. Constitutional Law for a Changing America: A Short Course. 5a ed. Thousand Oaks: CQ Press College, 2011.

EPSTEIN, Richard A. How Progressives Rewrote the Constitution. Washington: Cato Institute, 2007.

. The Classical Liberal Constitution: The Uncertain Quest for Limited Government. $1^{a}$ ed. Cambridge: Harvard University Press, 2017.

FERREIRA NETO, Arthur. O Originalismo na América. Cadernos do Programa de Pós-Graduação em Direito - PPGDir./UFRGS, n. 4, set. 2014. Disponível em: https://seer.ufrgs.br/ppgdir/article/view/50462. (acessado em 03/05/2019).

FRIEDMAN, Lawrence M. A History of American Law. $3^{\text {a }}$. ed. New York: Touchstone, 2005 .

GADAMER, Hans-Georg. Verdade e Método I. 15a . ed. Petrópolis: Vozes, 2016.

GORSUCH, Neil. A Republic, if You Can Keep It. New York: Crown Forum, 2019.

GERHART, Peter M. The Supreme Court and Antitrust Analysis: The (Near) Triumph of the Chicago School. The Supreme Court Review, 1982. pp. 319-349. Disponível em https://scholarlycommons.law.case.edu/faculty_publications/602, acessado em 05.07.2020.

GREEN, Christopher R., Originalism and the Sense-Reference Distinction. St. Louis University Law Journal, V. 50, 2006. Disponível em https://ssrn.com/abstract=798466, acessado em 18.06.2020.

GREENE, Jamal, On the Origins of Originalism. Texas Law Review, Vol. 88; Columbia Public Law Research Paper No. 09-201, 2009. Disponível em: https://ssrn.com/abstract=1357541 (acessado em 25/07/2018).

GREY, Thomas C.. Origins of the Unwritten Constitution: Fundamental Law in American Revolutionary Thought. Stanford Law Review Vol. 30, No. 5, 1978. 
Disponível em https://www.jstor.org/stable/1228166 (acessado em 28/05/2018).

HAMILTON, Alexander; JAY, John; MADISON, James. The Federalist Papers. New York: Signet Classics, 2003.

HAYWARD, Stefen F. Two Kinds of Originalism. National Affairs n. 30, 2017. Disponível em http://www.nationalaffairs.com/publications/detail/two-kinds-oforiginalism (acessado em 24/01/2017).

HORNBY, A. S. Oxford Advanced Learner's Dictionary. $6^{\text {a }}$ ed. Oxford: Oxford University Press, 2000.

HUSCROFT, David; MILLER, Bradley W. (Org.). The Challenge of Originalism. $1^{\mathrm{a}}$ ed. New York: Cambridge University Press, 2011.

KAVAnAugh, Brett M. Our Anchor for 225 Years and Counting: The Enduring Significance of the Precise Text of the Constitution. Notre Dame Law Review, n. 89, 2014. Disponível em https://scholarship.law.nd.edu/ndlr/vol89/iss5/1, acessado em 08.07.2020.

KAY, Richard. "Originalist" Values and Constitutional Interpretation. Faculty Articles and Papers. 93, 1996. Disponível em https://opencommons.uconn.edu/law_papers/93 (acessado em 05/07/2018).

LAWSON, Gary. On Reading Recipes... and Constitutions. Georgetown Law Journal, n. $85, \quad$ pp. 1823-1836, $1997 . \quad$ Disponível em https://heinonline.org/HOL/LandingPage?handle=hein.journals/glj85\&div=49\&id=\&pa ge $=$, acessado em 25/04/2018.

. On Getting It Right: Remembering Justice Antonin Scalia. Boston University Law Review n. 299, 2016. Disponível em https://scholarship.law.bu.edu/faculty_scholarship/700/, acessado em 08.07.2020.

LEVY, Robert; MELLOR, William. The Dirty Dozen: How Twelve Supreme Court Cases Radically Expanded Government and Eroded Freedom. Washington: Cato Institute, 2010.

MALTZ, Earl M., Originalism and the Desegregation Decisions-A Response to Professor Mcconnell. Constitutional Commentary. 288. Disponível em https://scholarship.law.umn.edu/concomm/288 (acessado em 25/08/2018). 
MANNING, John F., Textualism and the Equity of the Statute. 101 Colum. L. Rev. 1, 2001. Disponível em SSRN: https://ssrn.com/abstract=2849561 (acessado em 01/06/2018).

. Textualism As a Nondelegation Doctrine. 97 Colum. L. Rev. 673, 1997.

Disponível em SSRN: https://ssrn.com/abstract=2849460 (acessado em 01/06/2018).

McCONNELL, Michael W.. Originalism and the Desegregation Decisions. 81 Virginia Law Review 947 (1995). Disponível em https://chicagounbound.uchicago.edu/cgi/viewcontent.cgi?article=12624\&context=jour

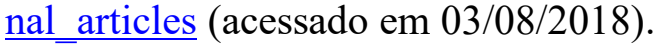

MEESE III, Edwin. Speech Before the BAR Association, in: CALABRESI, Steven G. (org.). Originalism - A Quarter-Century of Debate. Washington: Regnery, 2007. pp. 47-54.

MERRILL, Thomas W. Originalism, Stare Decisis and the Promotion of Judicial Restraint. Constitutional Commentary n. 1092, 2005. Disponível em https://scholarship.law.umn.edu/concomm/1092 (acessado em 03/07/2018).

MONAGHAN, Henry P.. Our Perfect Constitution. 56 N.Y.U. L. Rev. 353, 1981.

Dsiponível em: https://scholarship.law.columbia.edu/faculty_scholarship/778 (acessado em 18/06/2018).

MORAES, Alexandre de. Jurisdição Constitucional e Tribunais Constitucionais. $3^{\text {a }}$ ed. São Paulo: Atlas, 2013.

MORALES, Cesar Mecchi. Originalismo e Interpretação Constitucional. (Tese de Doutorado). São $\quad$ Paulo, 2011. Disponível em http://www.teses.usp.br/teses/disponiveis/2/2134/tde-16042012-161140/pt-br.php, acessado em 12/12/2016.

O'NEILL, Johnathan. Originalism in American Law and Politics: A Constitutional History. Baltimore: The Johns Hopkins University Press. Edição Eletrônica.

POST, Robert C.; SIEGEL, Reva B.. Originalism as a Political Practice: The Right's Living Constitution. Fordham Law Review, v. 75, 2006. Disponível em https://digitalcommons.law.yale.edu/fss_papers/171/, acessado em 21.05.2020.

POWELL, Haywood Jefferson. The Original Understanding of Original Intent. 
Harvard Law Review, Volume n. 98, n. 5, 1985. Disponível em: https://ssrn.com/abstract=1973768, acessado em 05/06/2018.

. Rules For Originalists. 73 Virginia Law Review 659-699, 1987. Dsiponível em https://scholarship.law.duke.edu/faculty_scholarship/444/ (acessado em 01/06/2018). PRIEST, George L.. The Abiding Influence of the Antitrust Paradox: An Essay in Honor of Robert H. Bork. Harvard Journal of Law and Public Policy, 31(2), 2008. pp. 455-463. Disponível em https://digitalcommons.law.yale.edu/fss_papers/643/, acessado em 06.07.2020.

RAKOVE, Jack N. (Org.). Interpreting the Constitution - The Debate over Original Intent. $1^{\text {a }}$ ed. Boston: Northeastern University Press, 1990.

. The Annotated U.S. Constitution and Declaration of Independence.

Cambridge: Harvard University Press, 2009.

RAMOS, Elival da Silva. Ativismo Judicial. 1ª ed. São Paulo: Saraiva, 2010.

RAPPAPORT, Michael B., Originalism and the Colorblind Constitution. San Diego Legal Studies Paper No. 13-115, 2013. Disponível em: https://ssrn.com/abstract=2244610 (acessado em 01/09/2018).

RAPPAPORT, Michael B.; MCGINNIS, John O.; SHAPIRO, Ilya; WALSH, Kevin C.; WURMAN, Ilan. The Legal Turn in Originalism: A Discussion. San Diego Legal Studies Paper No. 18-350, 2018. Disponível em: https://ssrn.com/abstract=3201200 (acessado em 30/07/2018).

RAPPAPORT, Michael B.; SMITH, Steven Douglas; BAUDE, William; SACHS, Stephen E. The New and Old Originalism: A Discussion San Diego Legal Studies Paper No. 15-178, 2014. Disponível em : https://ssrn.com/abstract=2562531 (acessado em 17/08/2018).

RAPPAPORT, Michael B.; McGINNIS, John O. Original Methods Originalism: A New Theory of Interpretation and the Case Against Construction (May 19, 2009). Northwestern University Law Review, Vol. 103, No. 2, 2009. Disponível em: https://ssrn.com/abstract=1407274 (acessado em 05/08/2018).

. Originalism and the Good Constitution. Cambridge: Harvard University Press, 2013. 
A Pragmatic Defense of Originalism. Northwestern University Law Review, Vol. 101, 2007. Disponível em: https://ssrn.com/abstract=956477 (acessado em 07/08/2018).

. Original Interpretative Principles as the Core of Originalism. San Diego Legal Studies Paper No. 07-82, 2007. Disponível em: https://ssrn.com/abstract=962142 (acessado em 08/08/2018).

. Our Supermajoritarian Constitution. Texas Law Review, v. 80, n. 4, 2002. Disponível em: https://ssrn.com/abstract=288344, acessado em 05.05.2020.

. Reconciling Originalism and Precedent. Northwestern University Law

Review, Vol. 103, No. 2, 2009. Disponível em: https://ssrn.com/abstract=1399504 (acessado em 05/07/2018).

REHNQUIST, William H.. The Supreme Court. New York: Vintage Books, 2002.

. The Notion of a Living Constitution. Texas Law Review, V. 54, n. 4, 1976. Disponível

em https://heinonline.org/HOL/LandingPage?handle=hein.journals/tlr54\&div=38\&id=\&pa ge=, acessado em 23.02.2020.

RING, Kevin A.. Scalia Dissents: Writings of the Supreme Court's Wittiest, Most Outspoken Justice. Washington: Regnery Publishing, 2016.

ROSTRON, Allen. Justice Scalia's Truthiness and the Virtues of Judicial Candor. Indiana Law Journal, n. 89, suplemento n. 12, 2013. Disponível em https://papers.ssrn.com/sol3/papers.cfm?abstract $\mathrm{id}=2373277$, acessado em 07.07.2020. SARMENTO, Daniel; SOUZA NETO, Cláudio Pereira de. Direito Constitucional Teoria, História e Método de Trabalho. $2^{\mathrm{a}}$ ed. Belo Horizonte: 2014.

SCALIA, Antonin. A Matter of Interpretation - Federal Courts and The Law. $1^{\text {a }}$ ed. Princeton: Princeton University Press, 1998.

. The Rule of Law as a Law of Rules. The University of Chicago Law Review, VOLUME 56 NUMBER 4 FALL, 1989. Disponível em http://chicagounbound.uchicago.edu/cgi/viewcontent.cgi? article $=4632 \&$ context $=$ uclrev (acessado em 24/01/2017).

; GARNER, Bryan A. Reading Law. $1^{\text {a }}$ ed. St. Paul: Thomsom/West, 2012. 
Versão eletrônica.

. Palestra proferida em 14.06.1986 no UNITED STATES DEPARTMENT

OF JUSTICE, OFFICE OF LEGAL POLICY. Original Meaning Jurisprudence: a sourcebook, $\quad$ n. $106,1987 . \quad$ Disponível em https://catalog.hathitrust.org/Record/002455032, acessado em 15.07.2020. pp. 101-106. SILVA, Virgílio Afonso da (Org.). Interpretação Constitucional. $1^{a}$ ed. São Paulo: Malheiros Editores, 2010.

SCHAUER, Frederick. Defining Originalism. 19 Harv. J. L. \& Pub. Pol'y 343 (19951996). Disponível em https://heinonline.org/HOL/LandingPage?handle=hein.journals/ hjlpp19\&div $=32 \&$ id $=\&$ page $=($ acessado em 28/02/2018).

SOLUM, Lawrence B. Originalism and the Natural Born Citizen Clause. Illinois Public Law Research Paper No. 08-17, 2010. Disponível em https://ssrn.com/abstract=1263885 (acessado em 02/02/2017).

. A Reader's Guide to Semantic Originalism and a Reply to Professor

Griffin. Illinois Public Law Research Paper No. 08-12, 2008. Disponível em https://ssrn.com/abstract=1130665 (acessado em 02/02/2017).

. Semantic Originalism. Illinois Public Law Research Paper No. 07-24, 2008. Disponível em https://ssrn.com/abstract=1120244 (acessado em 02/02/2017).

. What is Originalism? The Evolution of Contemporary Originalist

Theory. 2010. Disponível em https://ssrn.com/abstract=1825543 (acessado em 24/01/2017).

SOLUM, Lawrence B.; BENNET, Robert W. Constitutional Originalism - A Debate. Ithaca: Cornell University Press, 2011.

SOLUM, Lawrence B.. The Constraint Principle: Original Meaning and Constitutional Practice. Disponível em: https://ssrn.com/abstract=2940215 (acessado em 07/08/2018).

. The Fixation Thesis: The Role of Historical Fact in Original Meaning. 91 Notre Dame Law Review 1, 2015. Disponível em: https://ssrn.com/abstract=2559701 (acessado em 02/08/2018).

. What is Originalism? The Evolution of Contemporary Originalist 
Theory, in HUSCROFT, David; MILLER, Bradley W. (Org.). The Challenge of Originalism. $1^{\mathrm{a}}$ ed. New York: Cambridge University Press, 2011. p. 12.

SOMIN, Ilya. The Borkean Dilemma: Robert Bork and the Tension between Originalism and Democracy. The University of Chicago Law Review Dialogue, Vol. 80, pp. 243-255, 2013. Disponível em https://lawreview.uchicago.edu/sites/lawreview. uchicago.edu/files/uploads/Dialogue/Somin Online.pdf\#page=1\&zoom=auto,-30,792 (acessado em 24/01/2017).

Originalism and Political Ignorance. Minnesota Law Review, Vol. 97, No. 2, pp. 625-668, December 2012; George Mason Law \& Economics Research Paper No. 12-28, 2012. Disponível em https://ssrn.com/abstract=2015006 (acessado em 03/02/2017).

STRANG, Lee J., An Originalist Theory of Precedent. New Mexico Law Review, Vol. 36, p. 419, 2006. Disponível em https://ssrn.com/abstract=772165 (acessado em $03 / 02 / 2018)$.

STRAUSS, David A.. The Living Constitution. New York: Oxford University Press, 2010 .

STRECK, Lenio Luiz. Hermenêutica Jurídica e(m) Crise. $10^{\mathrm{a}}$ ed. Porto Alegre: Livraria do Advogado, 2011.

SUNSTEIN, Cass R. Antonin Scalia, Living Constitutionalist. Harvard Law Review, Forthcoming; Harvard Public Law Working Paper No. 16-15, 2016. Disponível em https://ssrn.com/abstract=2759938 (acessado em 02/02/2017).

THAYER, James Bradley. Legal Essays. Boston: The Boston Book Company, 1908.

VIEIRA, Oscar Vilhena. Supremocracia. Revista Direito GV, jul./dez. 2008, p. 441-464. VOLLI, Ugo. Manual de Semiótica. 1ª ed. São Paulo: Edições Loyola, 2007.

WEST, Robin. Progressive and Conservative Constitutionalism. Michigan Law Review, v. 88, 1990. Disponível em https://scholarship.law.georgetown.edu/facpub/639/, acessado em 18.03.2020.

WHITTINGTON, Keith E. Constitutional Interpretation - Textual Meaning. Original Intent \& Judicial Review. Lawrence: University Press of Kansas, 1999. . Originalism, Constitutional Construction, and the Problem of Faithless 
Electors. Arizona Law Review, 59-4, 2017. Disponível em: https://ssrn.com/abstract=2927464 (acessado em 01/08/2018).

WURMAN, Ilan. A Debt Against the Living - An Introduction to Originalism. Cambridge: Cambridge University Press, 2017.

. The Original Understanding of Constitutional Legitimacy. Brigham Young University Law Review, 819, 2015. Disponível em: https://digitalcommons.law.byu.edu/lawreview/vol2014/iss4/3 $\quad$ (acessado em 28/06/2018). 